\title{
Facial Nerve Nucleus
}

National Cancer Institute

\section{Source}

National Cancer Institute. Facial Nerve Nucleus. NCI Thesaurus. Code C12898.

A collection of cell bodies of cranial nerve VII found in the brainstem. There are three nuclei with distinct functions: the facial motor nucleus, which sends muscular efferent nerves to the muscles of facial expression and the stapedius; the superior salivary nucleus, which sends visceral parasympathetic efferent nerves mostly to the lacrimal and salivary glands; and the geniculate gang lion, which receives mostly afferent nerves for taste sensation. 